\title{
Refractory Perianal HPV Infection Secondary to GATA2 Immunodeficiency: A Case Report in Brazil
}

\author{
Lucas Rodrigues Boarini*, Carlos Walter Sobrado, Dewton de Moraes Vasconcelos, Sérgio Carlos Nahas and \\ Ivan Cecconello \\ Departamento de Gastroenterologia, Universidade de São Paulo, Brazil \\ Submission: January 20, 2019; Published: February 06, 2020 \\ *Corresponding author: Lucas Rodrigues Boarini, Departamento de Gastroenterologia, Hospital das Clínicas da Faculdade de Medicina, Universidade \\ de São Paulo, Av. Dr. Enéas de Carvalho Aguiar, 255. Cerqueira César - São Paulo/SP 05403-000, Brazil
}

\begin{abstract}
Introduction: GATA2 immunodeficiency is a rare disease of autosomal dominant inheritance caused by a mutation in the GATA2 gene that is a hematopoietic transcriptional factor related to the differentiation of lymphoid and myeloid lineages. These patients commonly present severe perianal infection from the human papillomavirus (HPV) that is refractory to local clinical surgical treatment associated with lymphomyeloid changes.

Case Report: The patient is male, 46 years old, white, married presented multiple anal canal warts and anitis, presented leukopenia resulting from lymphopenia and an absence of monocytes. The patient underwent excision, electrocoagulation, and perianal lesion curettage, whose pathological evidence showed intraepithelial neoplasia secondary to HPV, initially treated with topical imiquimod, ciprofloxacin, and metronidazole, improving the intestinal condition, but worsening the perineal lesions. After a perianal surgical procedure, he presented difficulty in healing, a progression of the ulcerated area, evolving into fistulizing disease with an altering of the local epithelial aspect. During the course of the study, he had severe pneumonia three times, early and extensive recurrences of perianal HPV, underwent eight perianal respective procedures, and started hematologic analysis due to lymphopenia. Lymphocyte immunophenotyping showed lymphopenia B, partial T lymphopenia, and progression to associated NK lymphopenia. The patient evolved with severe pancytopenia consistently accompanied by monocytopenia on each occasion, thus being acquired myelodysplasia. Genetic sequencing for cellular immunodeficiency screening was performed, showing GATA2 deficiency. The patient was referred to the bone marrow transplant sector, but it could not be performed due to recurrent infections.
\end{abstract}

Conclusion: GATA2 deficiency is a newly discovered immunological disorder that may lead to complex perianal disease, secondary to HPV infections. Conventional surgical treatment for HPV leads to worsening of lesions, and allogeneic bone marrow transplantation is recommended, when possible.

Keywords: GATA2 deficiency; Immunodeficiency syndromes; HPV infection; Allogeneic transplantation; Condyloma acuminate; Monocytes; Genetic testing

Core Tip

GATA2 immunodeficiency is an autosomal dominant disease that usually presents itself with a diagnosis of genital HPV that is refractory to clinical surgical treatment. Early diagnosis is rarely achieved because it is a disease almost unknown to gastroenterologists and coloproctologists, although the initial symptoms are secondary to HPV. This case report not only illustrates perianal disease and demonstrates the immunological deficiencies of GATA2 immunodeficiency, but also applies genetic analysis for obtaining a diagnosis and advises on the appropriate treatment.

Abbreviations: HPV: Human Pappilomavirus; DNA: Deoxyribonucleic Acid; AIDS: Acquired Immune Deficiency Syndrome; NK: Natural Killer; HIV: Human Immunodeficiency Virus; PPD: Purified Protein Derivative; Hb: Hemoglobin; MDS: Myelodysplastic Syndromes; AML: Acute Myeloid Leukemia; NTM: Non-Tuberculous Mycobacteriosis; HLA: Human Leukocyte Antigen; XIAP: X-Linked Inhibitor of Apoptosis

\section{Introduction}

Human papillomavirus (HPV) is a highly contagious DNA virus that can present with benign and malignant perianal lesions. It has a prevalence of $26.8 \%$, being the most frequent sexually

transmitted disease [1]. Persistent infection and development of the clinical disease through genital warts and ulcerations occur more frequently in immunosuppressed patients [2]. Primary im- 
munodeficiencies are poorly known and difficult to diagnose but, AIDS, immunosuppression secondary to medications, and transplant patients are known risk factors for condylomatous lesions. GATA2 immunodeficiency is a rare disease of autosomal dominant inheritance caused by a mutation in the GATA2 gene that is a hematopoietic transcriptional factor related to the differentiation of lymphoid and myeloid lineages. Usually, patients have lymphopenia B, decreased natural killer (NK) cells and partially decreased $\mathrm{T}$ lymphocyte numbers, monocytopenia affecting monocyte-derived cells, lymphedema, and are susceptible to intracellular infections and hematopoietic myeloid neoplasms $[3,4]$. The genital HPV infections - prevalent in the disease (63\%) - are refractory to local treatment and have early recurrences [5]. These patients may have genital HPV infection as the first manifestation of the disease, whether or not associated with leukocyte changes (leukopenia and monocytopenia) at the onset of the disease. Coloproctologists, urologists, and gynecologists being unfamiliar with GATA2 immunodeficiency may result in patients being submitted to multiple respective surgical procedures and can lead to anatomical alterations of the anogenital region with complex functional consequences such as fecal incontinence and anal stenosis. For this reason, the objective of this paper is to report and illustrate the perianal involvement of HPV in a patient with GATA2 immunodeficiency, a newfound and little known entity, who most often presents HPV infection refractory to clinical surgical treatment, which is associated with immunological changes and severe systemic infections.

\section{Case Report}

The patient is male, 46 years old, white, married, and a systems analyst. In 2006 he presented a stage of acute mucosanguinolent diarrhea, approximately twelve episodes per day, associated with fever, colic pain, and abdominal distension, he also complained of penile wart injury. He was diagnosed with bacterial enterocolitis and dehydration and started treatment with ciprofloxacin and volume replacement therapy at an inpatient unit for 48 hours with good clinical evolution. He underwent an outpatient local excision of a verrucous lesion but had no record of the histological etiology of the lesion. In 2008, he complained of warts and anal itching. The proctological examination showed the presence of multiple perianal and anal canal warts and anitis, and Refractory Perianal HPV and Gata2 Immunodeficiency leukopenia with $2.91 \times 10^{3}$ leukocytes $/ \mathrm{mm}^{3}\left(4.0-11.0 \times 10^{3} / \mathrm{mm}^{3}\right)$ at the expense of lymphopenia and absence of monocytes (Table 1). After HIV negative serology testing, the patient underwent excision, electrocoagulation, and curettage of the perianal lesions, whose pathological evidence showed high-grade intraepithelial neoplasia secondary to HPV, other infectious diseases were ruled out by negative cultures and a nonreactive PPD skin test. The patient returned after three weeks with a worsening of perineal lesions and early recurrence, presented difficulty healing, and with an alteration of the local epithelial aspect and new warty lesions. He underwent topical imiquimod treatment for three months and underwent eight perineal procedures with curettage, excision, and debridement from 2008 to 2011, with local worsening.

Table 1: Main altered laboratorial patient's parameters.

\begin{tabular}{|c|c|c|c|c|}
\hline Parameters & $\mathbf{2 0 0 8}$ & $\mathbf{2 0 0 9}$ & $\mathbf{2 0 1 9}$ & Normal Value \\
\hline Hemoglobin & $8.6 \mathrm{~g} / \mathrm{dl}$ & $9.5 \mathrm{~g} / \mathrm{dl}$ & $7.9 \mathrm{~g} / \mathrm{dl}$ & $13-18 \mathrm{~g} / \mathrm{dl}$ \\
\hline Leukocytes & $2.91 \times 103 / \mathrm{mm}^{3}$ & $2.57 \times 103 / \mathrm{mm}^{3}$ & $1.33 \times 103 / \mathrm{mm}^{3}$ & $4-11 \times 103 / \mathrm{mm}^{3}$ \\
\hline Monocytes & 0 & 0 & 0 & $0.2-0, \times 103 / \mathrm{mm}^{3}$ \\
\hline Plaquetas & $72000 / \mathrm{mm}^{3}$ & $83000 / \mathrm{mm}^{3}$ & $66000 / \mathrm{mm}^{3}$ & $140-450 \times 103 / \mathrm{mm}^{3}$ \\
\hline Ly T (CD45) & 701 cells $/ \mathrm{mm}^{3}$ & 608 cells $/ \mathrm{mm}^{3}$ & $701 \mathrm{cells} / \mathrm{mm}^{3}$ & $990-3300 \mathrm{cells}^{3} / \mathrm{mm}^{3}$ \\
\hline LyB (CD45/CD19) & $2 \%$ & $2 \%$ & $0,38 \%$ & $(5-20 \%)$ \\
\hline NK Cells & $44 \%$ & $32 \%$ & $3,4 \%$ & $(4-28 \%)$ \\
\hline
\end{tabular}

He began hematological analysis in 2009 as a result of leukopenia with a bone marrow biopsy that showed normal cellularity and normal maturation for his age, lymphocyte immunophenotyping showed B cell 9 cells $/ \mathrm{mm}^{3}\left(72-520\right.$ cells $\left./ \mathrm{mm}^{3}\right)$, partial T lymphopenia CD45 608 cells $/ \mathrm{mm}^{3}$ (990-3300 cells $/ \mathrm{mm}^{3}$ ) and progression to associated NK dysregulation (Table 1). The patient chose to end treatment in 2011, as no improvement was identified. He returned to follow-up in 2018, during the last years he developed three severe bouts of pneumonia requiring hospitalization and a worsening of perianal lesions evolving into complex perineal disease (Figures $1 \& 2$ ). Perianal Crohn's disease was sus- pected for the complex local lesion and diarrheal episodes, but the hematological alteration did not corroborate the suspicion. He underwent a fistulotomy, excision, and curettage of perineal lesions, and was referred to the immunologist as a result of recurrent infections and severe pancytopenia ( $\mathrm{Hb} 7.9 \mathrm{~g} / \mathrm{dl}, 1330$ leukocytes/ $\mathrm{mm}^{3}$, and 66.000 platelets), accompanied by monocytopenia on each occasion, suspected Refractory Perianal HPV and Gata2 Immunodeficiency of associated myelodysplasia (table 1). The main etiological factor considered was the hypothesis of primary immunodeficiency. Genetic sequencing for cellular immunodeficiency (266 genes) screening was performed, showing a heterozygous 
variant c.1070C>T (p.Thr357Ile) GATA2 deficiency. The patient was then referred to the bone marrow transplant sector for the procedure in September 2019.
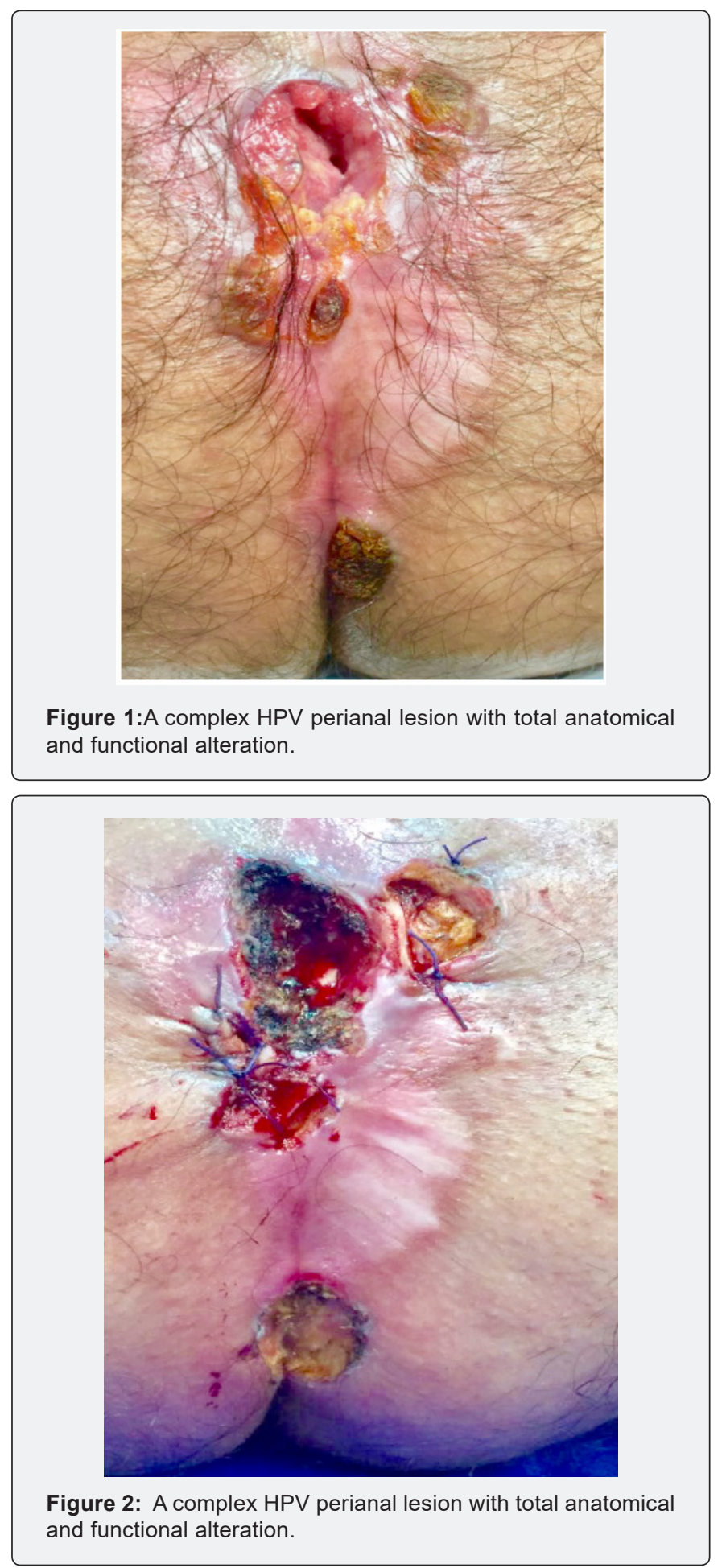

Discussion

GATA 2 hematopoietic transcription factor deficiency was described in 2011 and is responsible for the development of four previously described syndromes: monocytopenia and nontuber- culosis mycobacterial infection; [3,6] dendritic cell, monocyte, and NK cell deficiencies; [4,7] familial myelodysplastic syndromes (familial MDS)/acute myeloid leukemia (AML); [8] and Emberger syndrome (primary lymphedema and familial MDS) [9].

Clinical manifestations include immunodeficiency with marked susceptibility to human papillomavirus (HPV) infection, non-tuberculous mycobacteriosis (NTM), opportunistic fungal infection, a predisposition to familial MDS/AML, pulmonary alveolar proteinosis, and congenital lymphedema. Severe infections affect $82 \%$ of patients, with viral infections being the most common (70\%), we can highlight: dissemination of condylomatous lesions secondary to HPV (63\%) and severe herpetic infection (35\%) [5]. HPV immune protection requires cellular immunity related to CD4 T cells and NK cells. Decreasing NK cells in GATA2 immunodeficiency explains the spread of HPV [10]. An HPV infection is usually the first manifestation and typically refractory to local treatment (surgical excision, trichloroacetic acid, podophyllin, imiquimod) [5]. The patient described above has perianal HPV with high-grade dysplasia that is refractory to clinical surgical treatment. NTM may be present in $53 \%$ of the cases. For this reason, the culture of the material dried during the perineal surgical procedure is of fundamental importance. Bacterial infections may be present in $49 \%$ of patients, the most frequent being bacteremia (21\%), skin and subcutaneous tissue infection (19\%), pneumonia (14\%), and colitis (9\%) [5]. The patient in question had three severe bouts of pneumonia and enterocolitis. The presence of severe and recurrent HPV perianal infection, excluding known predisposing factors (HIV, transplantation, and drug immunosuppression), should raise suspicion for primary immunodeficiencies, such as GATA2 deficiency. Lack of knowledge on the immunological disease and a delay in initiating the correct treatment or administering inadequate treatment may lead to irreversible changes in the perineal region, such as anal stenosis or fecal incontinence due to chronic inflammation and multiple surgical procedures. The evolution into complex perianal disease leads to high morbidity and a negative impact on the patients' quality of life due to constant discharge of purulent secretion, impairment of the sphincter, and considerable recurrence rates despite the introduction of the correct treatment [2]. The primary and most studied systemic entity responsible for complex perianal disease is Crohn's disease, but there are other primary immunodeficiencies such as XIAP deficiency, as well as infectious diseases that have complex perianal lesions $[2,5,11]$ and that enter as differential diagnoses of GATA2 deficiency. The patient in question began showing perianal symptoms prior to the first report in the GATA2 immunodeficiency literature. Therefore, he underwent conventional treatment for HPV with multiple respective surgical procedures, and topical treatment with imiquimod, but with early and recurrent relapses that evolved into destruction of the sphincter and cutaneous deformity. Allogeneic transplantation from an HLA-compatible donor is still the best treatment for GATA2 deficiency. A study initially evaluating six patients undergoing allogeneic transplantation for 
GATA2 syndrome resulted in success in $83 \%$ of cases, with the removal of all immunosuppressive and antimycobacterial drugs, the absence of new infections, and normalization of immunological alterations [12]. However, this same group, when increasing the sample to 14 patients, observed a decrease in transplant response to $57 \%$ of the cases with an average follow-up time of 3.5 years and overall mortality in $42 \%$ of the cases. Two patients rejected the donor cells, and one patient relapsed with myelodysplastic syndrome [13]. These data confirm that although allogeneic transplantation achieves immune reconstitution, correction of susceptibility to infections, and reversal of propensity for myeloid progression, it still has a high overall mortality rate. In conclusion, GATA2 deficiency is a newly discovered immunological disorder that may lead to complex perianal disease, secondary to HPV infections. Conventional surgical treatment for HPV leads to a worsening of lesions, and allogeneic bone marrow transplantation is recommended, when possible.

\section{Declarations}

\section{Ethics approval and consent to participate}

This case report was submitted to the ethical committee Comitê de Ética para Análise de Projetos de Pesquisa (CAPPESQ) of the Hospital das Clínicas - Faculdade de Medicina and to Plataforma Brasil, with number approval 24378119.0.0000.0068.

\section{Competing Interests and Fundings}

The authors declare that this article doesn't have any competing interest and did not receive any funding specifically intended to accomplish this article.

\section{Availability of Data and Materials}

Data sharing is not applicable to this article as no datasets were generated or analysed during the current study, because it is a case report in which all relevant exams and patient's information are available in the article itself.

\section{Author's Contribution}

Lucas Rodrigues Boarini gathered and rewied the clinical data of the case report and wrote this article.

Carlos Walter Sobrado Junior wrote this article and was responsible for the treatment of the patient, especially for the surgical approach.

Dewton de Moraes Vasconcelos rewied this article and was responsible for the treatment of the patient, mainly the immunological approach.

Sérgio Carlos Nahas reviewed this article and was responsible for provide part of the institutional resources as a chief of the Colon and Rectal Surgery Division.
Ivan Cecconello reviewed this article and was responsible for provide part of the institutional resources as a chief of the Gastroenterology Department.

\section{References}

1. Dunne EF, Unger ER, Sternberg M, McQuillan G, Swan DC, et al. (2007) Prevalence of HPV infection among female in the United States. JAMA 297(8): 813-819.

2. Levi JE, Kleter B, Quint WGV, Fink MC, Canto CL, et al. (2002) High Prevalence of Human Papillomavirus (HPV) Infections and High Frequency of Multiple HPV Genotypes in Human Immunodeficiency Virus-Infected Women in Brazil. J Clin Microbiol 40(9): 3341-3345.

3. Vinh DC, Patel SY, Uzel G, Anderson VL, Freeman AF, et al. (2010) Autosomal dominant and sporadic monocytopenia with susceptibility to mycobacteria, fungi, papillomaviruses, and myelodysplasia. Blood 115(8): 1519-1529.

4. Bigley V, Haniffa M, Doulatov S, Wang XN, Dickinson R, et al. (2011) The human syndrome of dendritic cell, monocyte, B and NK lymphoid deficiency. J Exp Med 208(2): 227-234.

5. Spinner M, Sanchez LA, Hsu AP, Shaw PA, Zerbe CS, et al. (2014) GATA2 deficiency: a protean disorder of hematopoiesis, lymphatics and immunity. Blood 123(6): 809-821.

6. Hsu AP, Sampaio EP, Khan J, Calvo KR, Lemieux JE, et al. (2011) Mutations in GATA2 are associated with the autosomal dominant and sporadic monocytopenia and mycobacterial infection (MonoMAC) syndrome. Blood 118(10): 2653-2655.

7. Dickinson RE, Griffin H, Bigley V, Reynard LN, Hussain R, et al. (2011) Exome sequencing identifies GATA-2 mutation as the cause of dendritic cell, monocyte, B and NK lymphoid deficiency. Blood 118(10): 26562658.

8. Hahn CN, Chong CE, Carmichael CL, Ella J. Wilkins, Peter J. Brautigan, et al. (2011) Heritable GATA2 mutations associated with familial myelodysplastic syndrome and acute myeloid leukemia. Nat Genet 43(10): 1012-1017.

9. Mansour S, Connell F, Steward C, Ostergaard P, Brice G, et al (2010) Lymphoedema Research Consortium. Emberger syndrome-primary lymphedema with myelodysplasia: report of seven new cases. Am J Med Genet A 152(9): 2287-2296.

10. Mace EM, Hsu AP, Monaco-Shawver L, Makedonas G, Rosen JB, et al. (2013) Mutations in GATA2 cause human NK cell deficiency with specific loss of the CD56(bright) subset. Blood 121(14): 2669-2677.

11. Schwerd T, Pandey S, Yang HT, Bagola K, Jameson E, et al. (2017) Impaired antibacterial autophagy links granulomatous intestinal inflammation in Niemann-Pick disease type C1 and XIAP deficiency with NOD2 variants in Crohn's disease. Gut 66(6): 1060-1073.

12. Cuellar-Rodrigues J, Gea-Banacloche J, Freeman AF, Hsu AP, Zerbe CS, et al. (2011) Successful allogeneic hematopoietic stem cell transplantation for GATA2 deficiency. Blood 118(13): 3715-3720.

13. Grossman J, Cuellar-Rodrigues J, Gea-Banacloche J (2014) Nonmyeloablative allogeneic hematopoietic stem cell transplantation for GATA2 deficiency. Biol Blood marrow transplant 20(12): 19401948. 
This work is licensed under Creative Commons Attribution 4.0 License

DOI: 10.19080/ARGH.2020.14.555893

\section{Your next submission with JuniperPublishers} will reach you the below assets

- Quality Editorial service

- Swift Peer Review

- Reprints availability

- E-prints Service

- Manuscript Podcast for convenient understanding

- Global attainment for your research

- Manuscript accessibility in different formats ( Pdf, E-pub, Full Text, audio)

- Unceasing customer service

Track the below URL for one-step submission https://juniperpublishers.com/online-submission.php 